\title{
Programmable reference parameters for resonance locking in electro-thermal piezoresistive cantilever sensors
}

\author{
$\underline{\text { Andi Setiono }^{1,2}}$, Michael Fahrbach $^{1}$, Jiushuai Xu ${ }^{1}$, Wilson Ombati Nyang'au ${ }^{1,3}$, Maik Bertke ${ }^{1}$, \\ Hutomo Suryo Wasisto ${ }^{1}$, Erwin Peiner ${ }^{1}$ \\ ${ }^{1}$ Institute of Semiconductor Technology (IHT) and Laboratory for Emerging Nanometrology (LENA), \\ Technische Universität Braunschweig, Hans-Sommer-Straße 66, D-38106 Braunschweig, Germany \\ ${ }^{2}$ Research Centre for Physics, Indonesia Institute of Sciences (LIPI), Kawasan Puspitek Serpong, \\ 15314 Tangerang Selatan, Indonesia \\ ${ }^{3}$ Department of Metrology, Kenya Bureau of Standards (KEBS), Nairobi 00200, Kenya \\ a.setiono@tu-braunschweig.de,m.fahrbach@tu-braunschweig.de, jiushuai.xu@tu-braunschweig.de, \\ wilombat@tu-braunschweig.de,m.bertke@tu-braunschweig.de,h.wasisto@tu-braunschweig.de, \\ e.peiner@tu-braunschweig.de
}

\begin{abstract}
Resonant-frequency tracking is essential when measuring analyte targets using resonance-based sensors in real-time. We developed a self-sensing and -actuating silicon cantilever resonator that can be functionalized as an environmental sensor for analytes such as particles, humidity, and gases. However, direct thermal parasitic causes the sensor to generate a non-ideal output signal which hinders implementing a real-time phase-locked loop (PLL) based setup. To alleviate these problems, software de-embedding solutions to create an optimized signal, especially a monotonic phase response, are investigated. The de-embedding approach is based on subtracting a selectable reference, which mimics the parasitic behavior, from the measured data. All reference calculations are performed real-time on a microcontroller, which allows for on-line PLL based frequency tracking. Experiment results show a good response in resonant-frequency locking and tracking under exposure to cigarette smoke.
\end{abstract}

Keywords: De-embedding, programmable reference, electro-thermal piezoresistive cantilever sensor, monotonic phase response, phase-locked loop.

\section{Introduction}

Environmental health has been a concern of all parties globally in the past decades, which further has led to the development of environmental monitoring technology. Sensor technology is an important part of environmental monitoring, i.e., to perform one or more measurements that are necessary to assess the status of an environment. Measurements of particles, toxic gases, and volatile organic compounds (VOCs) are a part of the monitoring systems to protect persons against environmental deterioration. Nowadays, environmental monitoring emerges with the development of microsensors and microactuators in the field of micro-electromechanical system (MEMS) technologies. There are many environmental sensors available already, such as particle-, and gassensors. Most frequently, they are based on optical scattering and dispersion [1, 2]. Compared to optical-based sensor, MEMS resonance-based sensors have advantages in size, energy consumption, and high-volume fabrication. Electro-thermal piezoresistive cantilever sensors (EtPCS) are MEMS-based sensor-actuator systems that can reliably measure environmental conditions at high resolution [3-5]. Electro-thermal based actuation system has advantages in low fabrication complexity and high responsivity [6]. Furthermore, utilizing silicon piezoresistors as a sensing part results in high signal-to-noise ratio, high miniaturization capability, and compatibility with available electronics. Nevertheless, parasitic factors inside of the EtPCS are a drawback that has to be eliminated. Thermal parasitics can generate a Fano resonance (asymmetric shape) and a reversing phase response at the sensor output. A reversing phase response is an inhibitive factor in PLL-based real-time measurements. Therefore, the aim of this work is to optimize the phase response of EtPCS in order to work properly in a real-time measurement setup, i.e., by de-embedding the signal of the sensor using an approximation of the parasitic signal, 
which is subtracted from the measured signal. This approximated parasitic is also called reference.

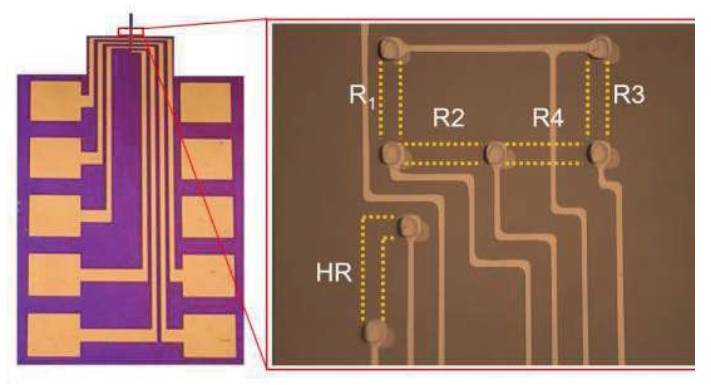

Fig. 1: Photograph of a MEMS electrothermal cantilever sensor and optical-micrograph magnification of the cantilever top surface showing the heating resistor $(H R)$ and the $U$ shape Wheatstone bridge (WB).

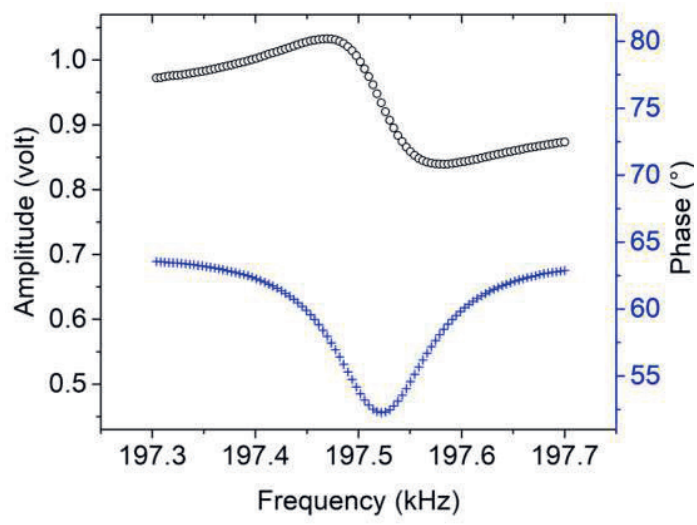

Fig. 2: $\quad$ Measured amplitude (black circle) and phase (blue plus signs) response of a piezoresistive cantilever excited by on-chip electrothermal actuation.

\section{Output characteristics of EtPCS}

We developed an electrothermal piezoresistive, i.e., a self-actuating and selfsensing, resonant cantilever (Fig. 1) for detecting various analyte targets. The main electrical components are a p-doped piezoresistive Wheatstone bridge (for sensing) and a $p$-doped heating resistor (for actuation). The heating resistor (HR) creates a mechanical actuation by converting thermal power through Joule heating [7]. This thermal power is generated by supplying the HR with an actuation signal consisting of a sine-wave $\left(V_{\mathrm{AC}} \sin (\omega t)\right)$ superimposed onto a constant voltage $\left(V_{D C}\right)$. The dynamic amplitude $V_{A C}$ leads to two frequencies being excited: $\omega$ and $2 \omega$. The constant component $V_{D C}$ creates constant stress on the cantilever beam. If $V_{D C}$ is much higher than $V_{\mathrm{AC}}$, the cantilever beam is driven into vibration at $\omega$, as the frequency component of $2 \omega$ is negligible. Here, the main component of the output signal has the same frequency as the excitation signal. The generated excitation force is then detected by the WB and converted into an electrical signal. However, direct thermal coupling exposed from the HR to the WB, creates a parasitic effect on the sensor output that induces an asymmetric resonance line-shape (Fano resonance) and phase deformation as shown in Fig. 2. The reversing phase response presents an ambiguity and is detrimental for real-time operation using a phase-locked loop (PLL) [8]. Therefore, there is a need for optimizing the amplitude and the phase response. This can be done by creating an approximation of the parasitic, also called reference, and subtracting it from the measured values. This way the ideal signal of the sensor is de-embedded from the measured signal.

\section{Reference signal subtraction}

Signal subtraction is intended to eliminate the parasitic parameters inside the EtPCS. In [8], Bertke et al. illustrated how a Fano resonance results from a superposition of a constant amplitude signal with Lorentzian-line-shape signal. Therefore, subtracting a constantamplitude signal from the output of the EtPCS is expected to reveal a Lorentzian amplitude shape and optimize the sensor phase response.

In previous work [9, 10], a system based on reference-signal subtraction for eliminating the parasitic effect and optimizing the EtPCS has been realized. The reference signal was created using passive electronic components to build an RCL (Resistor, Capacitor, Inductor) filter. Through this approach, symmetrical amplitude shapes and monotonic phase responses were demonstrated. However, as passive references require much effort to be tuned, a programmable reference is more desirable to be used. Thus, the current study seeks to implement a programmable reference as schematically shown in Fig. 3. The proposed instrument comprises three main parts, i.e., a cantilever sensor (EtPCS), an electronic measurement system, and a display and acquisition system. The electronic measurement system has bi-directional capability for communicating and commanding between the sensor and the display and acquisition system. It generates a sinusoidal signal through a wave generator component, which subsequently actuates the cantilever beam. It also transmits the measured data and 
de-embedding results to the acquisition system.

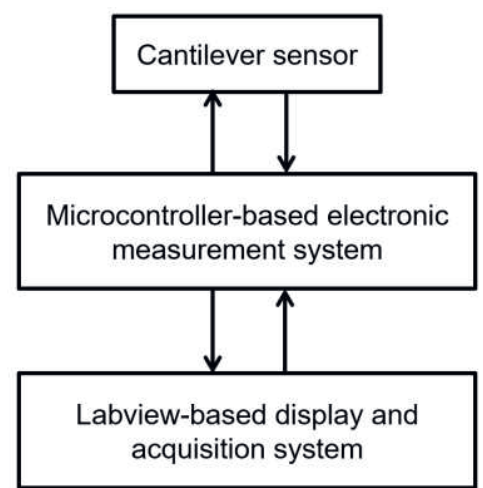

Fig. 3: Block diagram of the deembedding microcontroller system that has bi-directional communication for actuating and sensing of the cantilever sensor and further display and acquisition using a LabVIEW-based system.

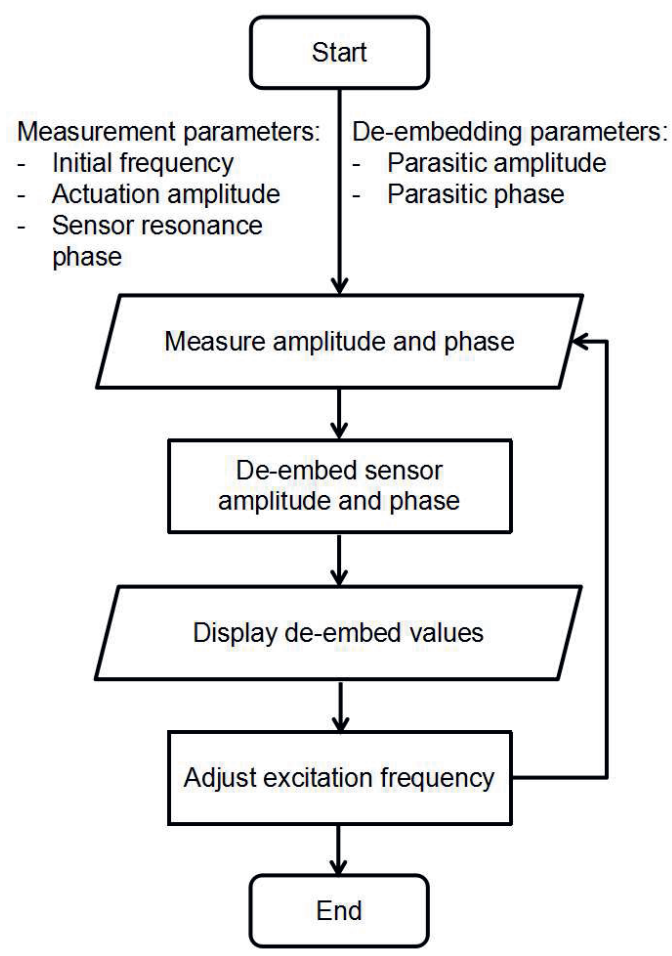

Fig. 4: Flowchart of de-embedding sensor signals using reference parameters which are transmitted to a microcontroller.

According to the flowchart delineated in Fig. 4, a microcontroller acts as a central processor for reading, processing and transmitting data. It measures the cantilever output signal amplitude, and simultaneously calculates the phase difference between the actuation and sensing signals. Afterwards, these sensor responses are plotted on a LabVIEW-based display system. On the other hand, reference parameters (i.e., amplitude and phase) are constant values, which are sent to the microcontroller by the LabVIEW system. Reference parameters are determined by considering the baselines of both amplitude and phase responses in the range of the working frequency (near resonance) [9, 11], as shown in Figs. $5 \mathrm{a}$ and $5 \mathrm{~b}$. These values are then used to de-embed the signal of the sensor from the measured signal. The corresponding formula is described in more detail in [11]. The resulting cantilever response shows optimized parameters (i.e., a nearly symmetrical amplitude shape and a monotonic phase response), as depicted in Fig. 5c. The resultant monotonic phase response can now be used in a PLL-based setup. All measurement and calculation results (i.e., amplitude and phase) are latterly captured on the display and acquisition system. In addition, the occurrence of the double peak might be avoided by implementing a lock-in amplifier principle that calculate the phase by using a DC component as a result of signal mixing.

\section{PLL-based frequency locking}

A monotonic phase response is needed for stable operation of a PLL-based system. With a monotonic phase response, the PLL can lock the resonance phase $\left(\varphi_{0}\right)$ at a certain value of frequency, which is subsequently interpreted as resonant frequency $\left(f_{r}\right)$. A block diagram of PLL-based frequency locking is shown in Fig. 6 , which consists of a phase detector, an Icontroller, and a feedback system. These three main elements are represented by the microcontroller in software. The phase detector measures the phase between the actuation signal and the output of the sensor, which is achieved by measuring the difference in time between the zero crossings of both signals. This time difference is proportional to the phase. The measured phase value is then used to calculate the de-embedded phase of the sensor. Afterwards, the phase is used by the I-controller for tracking the resonance phase. The I-controller is formulated by:

$f_{r}(t)=f_{0}+K_{i} \int_{0}^{t} \Delta \varphi_{\text {set }}(\tau)-\Delta \varphi_{\text {meas }}(\tau) d \tau$

where $f_{0}, K_{\mathrm{i}}, \varphi_{\text {set }}, \varphi_{\text {meas }}, t, \tau$ are initial frequency, integral coefficient, set-point phase, measured phase, time, and variable of integration, respectively.

The flowchart shown in Fig. 7 illustrates how the PLL operates to lock the resonant frequency. In the beginning, a desired phase is 


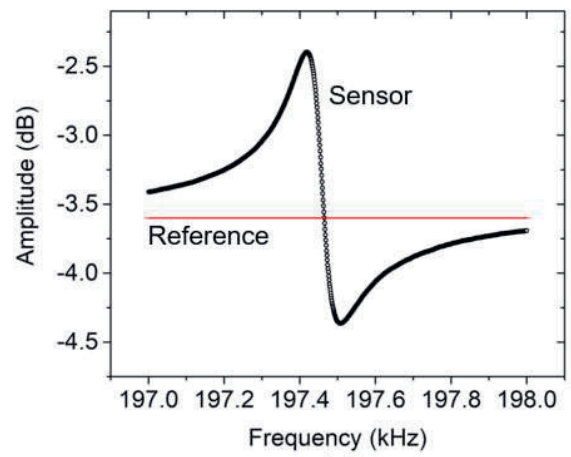

(a)

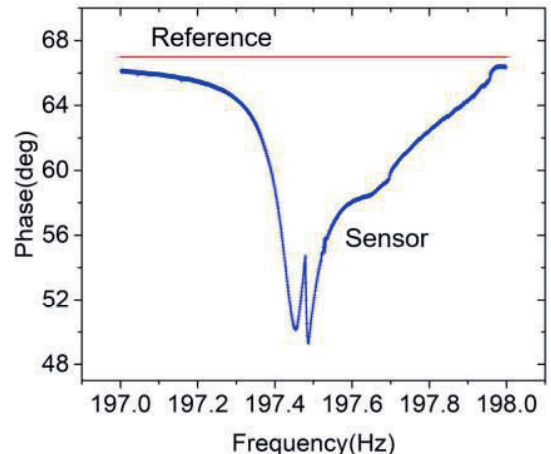

(b)

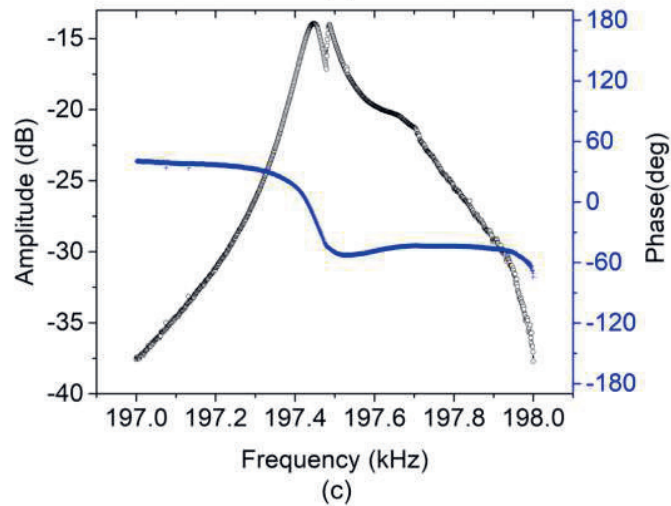

Fig. 5: Measured sensor signals. (a) Asymmetric amplitude response. (b) and reversed phase response. The occurrence of the double peak is expected due to losing time in phase calculation during a short resonance transition. (c) Amplitude (black circle line) and phase (blue plus line) responses with programmable references in place. De-embedding results in symmetrical amplitude shape and nearly monotonic phase response.

set as a set-point, which is related to the resonance phase of the sensor. Hereinafter, the microcontroller measures the phase between the sensor output and actuation signal and uses it in the de-embedding process. The resulting phase is then employed 
by the l-controller for adjusting the excitation frequency as described by Eq. (1).

Figure 8 shows that the de-embedded phase response can be used to track the resonant frequency. Increasing the integral coefficient $\left(K_{i}\right)$ of the I-controller leads to a decrease of response time, but on the other hand, an overshoot is generated. Therefore, an optimum value of $K_{\mathrm{i}}$ is needed for obtaining the locked condition as fast and precise as possible.

Furthermore, the EtPCS is investigated under slight exposure to cigarette smoke in a chamber as illustrated in Fig 9a. Frequency tracking uses the de-embedded sensor signal using the PLL-configuration shows good results as depicted in Fig 9b. In section $A$, the baseline of the resonant frequency was measured, showing a decreasing trend. The suspected reason is an increase in temperature inside the chamber. The temperature coefficient of Young's modulus and the thermal expansion coefficient are the intrinsic parameters of silicon that contribute most to its fast and reversible temperature response. As a confirmation, stability tests were conducted by using an MFLI Lock-in Amplifier (Zurich Instruments) under temperature and humidity conditions of $23.15 \pm 0.17^{\circ}$ and $38.5 \pm 0.7 \%$. The result shows a stable resonant frequency of $197405.05 \pm 0.01 \mathrm{~Hz}$ and $197405.16 \pm 0.06 \mathrm{~Hz}$ during 5 minutes and 1 hour of measurement duration, respectively.

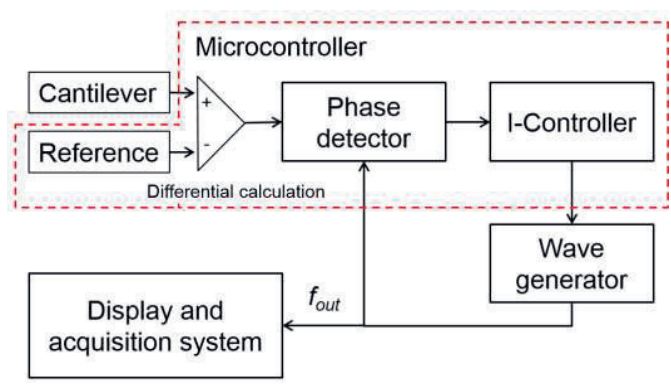

Fig. 6: A block diagram of the programmable reference subtraction in a PLL configuration.

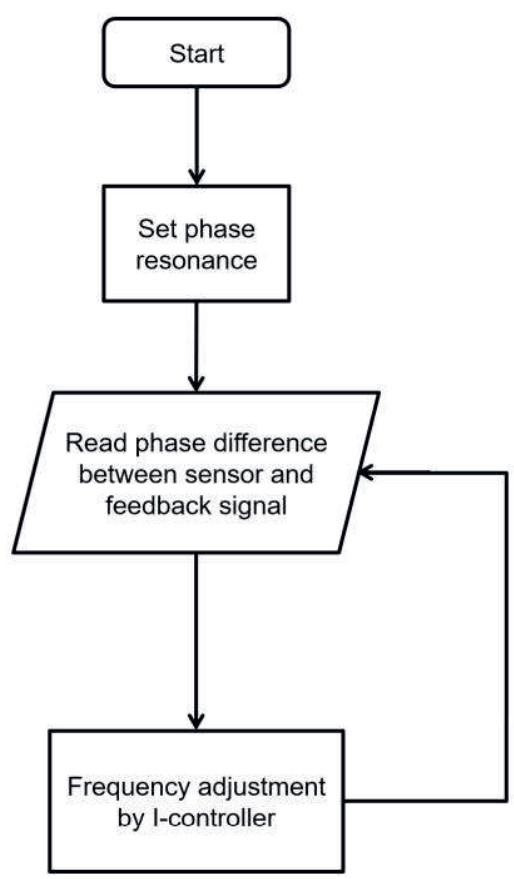

Fig. 7: Flowchart of the digital l-controller for managing a frequency actuation based on a phase error.

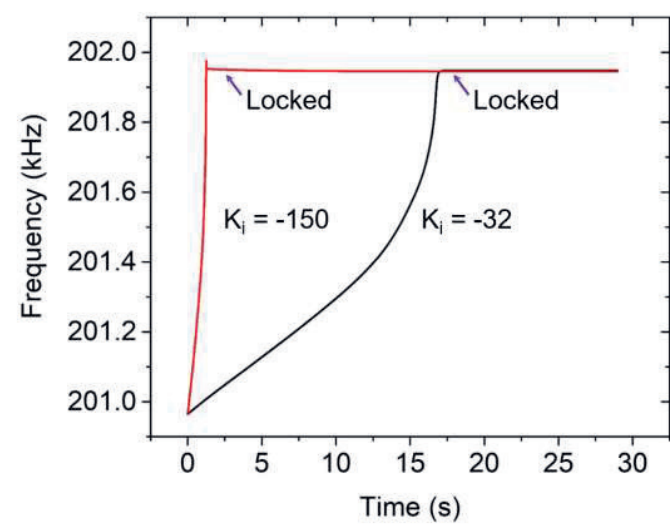

Fig. 8: Time-dependent resonance locking under a PLL set-up and an I-controller with $K_{\mathrm{i}}=-150 \mathrm{~Hz} / \%$ (red) and $K_{\mathrm{i}}=-32 \mathrm{~Hz} / \mathrm{s}$ (black). 


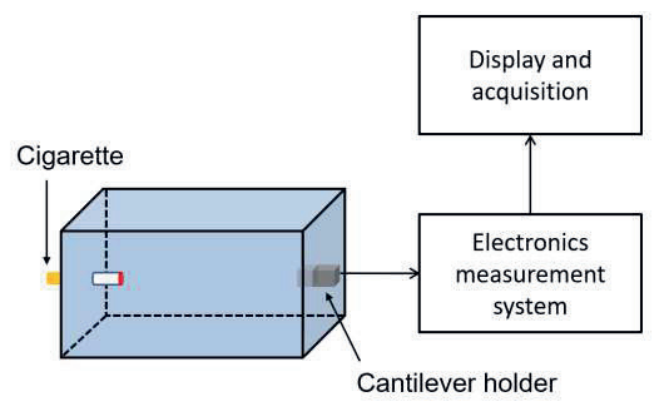

(a)

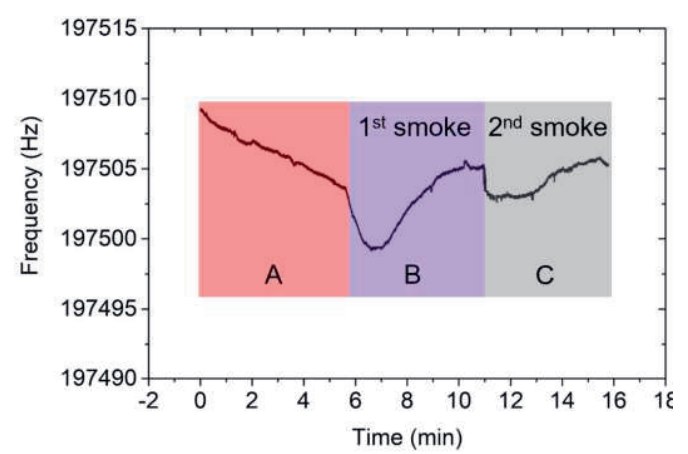

(b)

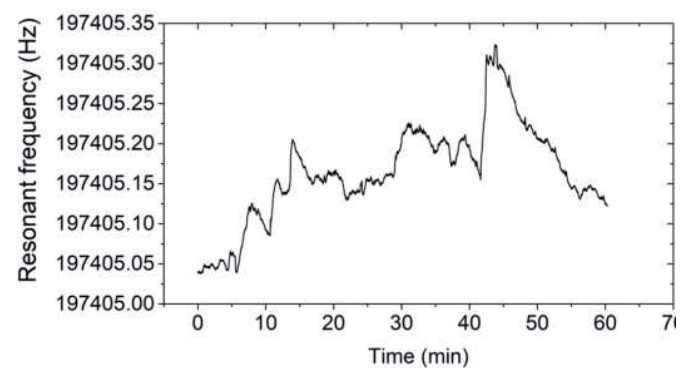

(c)

Fig. 9: (a). Measurement setup for realtime measurements of an EtPCS exposed to cigarette smoke. Timedependent resonant frequency using an electro-thermal piezoresistive cantilever sensor (b) under temperature changing and smoke exposing and (c) stability tests under relatively constant of temperature and humidity.

Furthermore, in the beginning of sections B and $C$ respectively, a burning cigarette was introduced into the chamber. The frequency drops in the beginning of each smoke exposure with the response times of $41.8 \mathrm{~s}$ and $2.8 \mathrm{~s}$ for the $1^{\text {st }}$ smoke and the $2^{\text {nd }}$ smoke, respectively. The ensuing responses show an act of recovering that is caused by transient loss of deposited cigarette smoke constituents from the resonator by evaporation [12]. The sensor shows a good recovery under exposure to cigarette smoke with recovery times of $126 \mathrm{~s}$ and $129.5 \mathrm{~s}$ for the $1^{\text {st }}$ smoke and the $2^{\text {nd }}$ smoke, respectively. However, a wet sensor cleaning by immersing in a special solution (e.g., acetone, isopropanol) [12-14] is still required to obtain optimal initial conditions. This method has even shown possibility to completely regenerate a cantilever sensor that has been fully loaded by nanoparticle agglomerates leading to instability during its operation [15].

\section{Conclusions}

The presented programmable de-embedding scheme works out properly with the reversing phase responses caused by crosstalk between heating resistor and piezoresistor. It provides flexibility in determining the reference parameters that latterly yields a monotonic phase response and demonstrate good frequency tracking under cigarette smoke exposure. The microcontroller has response time of $10 \mathrm{~ms}$ during measurements. In addition, the resolution is limited to ca. $0.1 \mathrm{~Hz}$ by the wave-generator. However, this programmable scheme requires a softwarePLL to be able to track the resonant frequency in real-time. Consequently, the main controller needs to have sufficient speed to calculate and execute the program more accurately measure the phase and de-embed the sensor signal. The controller used in this study can perform up to 64 million instructions per second. Further development and testing, however, are still needed, especially for calculating phase difference and providing a reference setup as quantitative comparison.

\section{Acknowledgments}

A. Setiono would like to thank the Ministry of Re-search, Technology and Higher Education of the Republic of Indonesia (RISTEKDIKTI) for the PhD scholarship of RISET-Pro under no. 343/RISET-Pro/FGS/VIII/2016 (World Bank Loan No. 8245-ID) which is revised by no. 37/RISET-Pro/FGS/III/2019 and IndonesianGerman Center for Nano and Quantum Technologies (IG-Nano) for the support. This work received funding from the European Union's Horizon 2020 research and innovation programme under no. 17IND05 MicroProbes. We are also grateful to Angelika Schmidt, Juliane Breitfelder, Aileen Michalski, KarlHeinz Lachmund, and Ratna Indrawijaya for their assistance during preparation of research tools as well as many fruitful discussions.

\section{References}

[1] W. Shao, H. Zhang, and H. Zhou, "Fine Particle Sensor Based on Multi-Angle Light Scattering and Data Fusion," (eng), Sensors (Basel, Switzerland), vol. 17, no. 5, 2017.

[2] Y. Wu, B. Yao, C. Yu, and Y. Rao, "Optical Graphene Gas Sensors Based on Microfibers: A Review," (eng), Sensors (Basel, Switzerland), vol. 18, no. 4, 2018.

[3] B. Sviličić, E. Mastropaolo, R. Zhang, and R. Cheung, "Tunable MEMS cantilever 
resonators electrothermally actuated and piezoelectrically sensed," Microelectronic Engineering, vol. 145, pp. 38-42, 2015.

[4] H. S. Wasisto, S. Merzsch, E. Uhde, A. Waag, and E. Peiner, "Handheld personal airborne nanoparticle detector based on microelectromechanical silicon resonant cantilever," Microelectronic Engineering, vol. 145, pp. 96-103, 2015.

[5] M. Bertke et al., "Strategy toward Miniaturized, Self-out-Readable Resonant Cantilever and Integrated Electrostatic Microchannel Separator for Highly Sensitive Airborne Nanoparticle Detection," (eng), Sensors (Basel, Switzerland), vol. 19, no. 4, 2019.

[6] HuiChao Shi, Shangchun Fan, Weiwei Xing, and Jinhao Sun, "2012 8th IEEE International Symposium on Instrumentation and Control Technology (ISICT): 11-13 July 2012, London, United Kingdom,"

[7] O. Brand, I. Dufour, S. M. Heinrich, and F. Josse, Resonant MEMS: Fundamentals, Implementation and Application / edited by Oliver Brand, Isabelle Dufour, Stephen M. Heinrich, and Fabien Josse.

Weinheim, Germany: Wiley-VCH, 2015.

[8] M. Bertke et al., "Analysis of asymmetric resonance response of thermally excited silicon micro-cantilevers for masssensitive nanoparticle detection," J. Micromech. Microeng., vol. 27, no. 6, p. 64001, 2017.

[9] A. Setiono et al., "Sensoren und messsysteme: Beiträge der 19. ITG/GMAFachtagung 26-27 Juni 2018 in Nürnberg,"

[10] A. Setiono et al., "Real-Time Frequency Tracking of an Electro-Thermal Piezoresistive Cantilever Resonator with ZnO Nanorods for Chemical Sensing," Chemosensors, vol. 7, no. 1, p. 2, 2019.

[11] A. Setiono et al., "Phase optimization of thermally actuated piezoresistive resonant MEMS cantilever sensors," J. Sens. Sens. Syst., vol. 8, no. 1, pp. 3748, 2019.

[12] J. Toledo et al., "Piezoelectric MEMS Resonators for Cigarette Particle Detection," (eng), Micromachines, vol. 10, no. 2, 2019.

[13] H. S. Wasisto et al., "Effect of Photoresist Coating on the Reusable Resonant Cantilever Sensors for Assessing Exposure to Airborne Nanoparticles," Procedia Engineering, vol. 47, pp. 302305, 2012.

[14] H. S. Wasisto et al., "Evaluation of photoresist-based nanoparticle removal method for recycling silicon cantilever mass sensors," Sensors and Actuators A: Physical, vol. 202, pp. 90-99, 2013.

[15] H. S. Wasisto, S. Merzsch, E. Uhde, A. Waag, and E. Peiner, "Partially integrated cantilever-based airborne nanoparticle detector for continuous carbon aerosol mass concentration monitoring," J. Sens. Sens. Syst., vol. 4, no. 1, pp. 111-123, 2015. 